\title{
Riesgo perioperatorio en pacientes con cirrosis hepática
}

\author{
MARIO CONCHA P., VERÓNICA MERTZ K.
}

\section{Perioperative risk among patients with cirrhosis}

Ten percent of cirrhotic patients with severely compromised liver function will require a surgical procedure. This article reviews the surgical risk associated with liver damage and surgery. The most important prognostic factor is the degree of functional impairment of the liver. It is evaluated using the Child-Pugh score, which has limitations that have been partially overcome by the Model for End Stage Liver Disease (MELD) score. Cardiac surgery has the highest risk, while extra-thoracic and extra-abdominal procedures have the lowest risk. The mortality for abdominal surgery fluctuates between 11 and 76\%. Biliary surgery is associated with frequent complications and mortality, which seem to decrease when the procedures are laparoscopic. There are few series that evaluate risk in Child $C$ patients. In liver resective surgery, liver function impairment and magnitude of the excision determine the risk. A high serum creatinine, cardiac failure and emergency surgery are independent risk factors. Although MELD score is useful to predict surgical risk, decision-making must be based on an individualized evaluation of each patient and careful planning of surgical procedures.

(Rev Med Chile 2010; 138: 1165-1171)

Key words: General surgery; Liver cirrhosis; Survival rate.

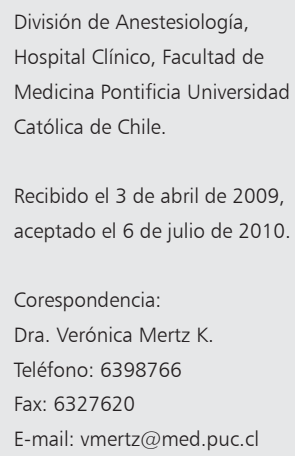<smiles>[Tl]</smiles>
a incorporación del trasplante hepático (TH) como una alternativa terapéutica aceptable en pacientes con cirrosis hepática $(\mathrm{CH})$, ha determinado una mejoría en las expectativas de sobrevida de estos pacientes. Como una de las consecuencias de ésto, muchos de ellos hoy son considerados y sometidos a procedimientos quirúrgicos mayores, cuyo riesgo, considerando el pronóstico de la $\mathrm{CH}$ y de la cirugía, era previamente considerado prohibitivo. Se estima que hasta $10 \%$ de los pacientes con $\mathrm{CH}$ requerirá cirugía cuando su función hepática ya se encuentre comprometida en forma importan$t^{1}$. Desafortunadamente, la cirugía y la anestesia comprometerán más aún una función hepática ya disminuida. Aún cuando no es el objetivo de esta revisión, se debe mencionar que factores como alteraciones del flujo sanguíneo hepático, efecto directo de drogas hepatotóxicas, sepsis, pueden explicar el deterioro funcional hepático que se observa en el post operatorio de los pacientes con
CH. De estos probablemente el más importante sea la disminución del flujo hepático como resultado de la anestesia y la cirugía ${ }^{2}$. Con frecuencia se ha atribuido una capacidad hepatotóxica directa de los agentes anestésicos inhalatorios (halotano, enflurano, isoflurano, sevoflurano, desflurano). Si bien estos son capaces de producir modificaciones de los niveles de transaminasas y fosfatasas, estos son leves y sin repercusión clínica. No existen evidencias que muestren un deterioro adicional de la función hepática secundario al uso de estos agentes. La temida necrosis hepática masiva producida por halotano, pero no exclusiva de este, es un cuadro diferente, de rara ocurrencia, con un mecanismo específico de producción, diferente al que determina las alteraciones mencionadas.

El deterioro de la función hepática que se agrega en el post operatorio plantea la necesidad de realizar una adecuada estimación del riesgo que enfrentan estos pacientes. En casos de pacientes candidatos o posibles candidatos a TH que requie- 
ren cirugía, el riesgo de ésta debe ser comparado con el de la postergación de la cirugía hasta después del TH. Desafortunadamente, la dificultad de realizar estudios adecuadamente controlados, diferencias en las definiciones utilizadas al evaluar morbilidad, forma de expresar los resultados, tipos de cirugía, la condición de cirugía electiva o de urgencia, y principalmente diferencias en el grado de funcionalidad hepática, determinan diferencias entre las distintas series reportadas. Todo esto dificulta la estimación de riesgo y la toma de decisiones. El objetivo de esta revisión fue evaluar la morbimortalidad perioperatoria de pacientes con $\mathrm{CH}$, sometidos a diversos tipos de cirugía.

\section{Método}

Se revisó la literatura a través de la base de datos Medline y su interfase Pubmed, Clinical Queries, usando "Liver Cirrhosis" asociado a las siguientes palabras: "Hepatectomy", "Cholecystectomy, Laparoscopic", "Carcinoma, Hepatocellular", "Surgical Procedures, Operative", "Cardiac Surgical Procedures", "Portasystemic Shunt, Transjugular Intrahepatic" "Mortality", "Prognosis", y "Hospital Mortality". La búsqueda se limitó a artículos publicados en inglés y español, en pacientes mayores de 18 años, en el período 1995 a 2009. Además de los artículos seleccionados se revisaron las bibliografías y artículos considerados relacionados, buscando responder preguntas acerca de la factibilidad de realizar cirugía en pacientes con distintos grados de compromiso funcional hepático, como se determina éste, cual es la morbimortalidad general y de algunos procedimientos específicos, que factores de riesgo son identificables, la importancia de su corrección, y oportunidad de la cirugía.

Numerosos estudios muestran que la cirugía es factible incluso en pacientes con grados avanzados de deterioro de la función hepática ${ }^{3-16}$. Se debería entonces tratar de precisar la morbimortalidad de cada cirugía en particular y en relación al grado de compromiso funcional hepático de cada paciente, determinar si existen condiciones de riesgo adicionales, si es factible reducir o eliminar el efecto de los factores de riesgo presentes $y$, finalmente, tratar de determinar si existe algún elemento clínico o de laboratorio que determine la factibilidad o contraindicación de la cirugía planificada.

\section{Determinación del grado de compromiso funcional}

La morbimortalidad reportada en las diferentes series está directamente relacionada con el grado de función hepática y con el tipo de cirugía realizada ${ }^{3-16}$. Aún cuando hay pocos estudios que comparen la morbimortalidad en pacientes con y $\sin \mathrm{CH}^{10,15,17}$, es un hecho de conocimiento general, que estos pacientes tienen un riesgo mayor de morbimortalidad perioperatoria, el cual aumenta en la medida que el deterioro de la función hepática progresa $a^{4-6,8,17}$. Se hace entonces evidente la importancia de la determinación del grado de compromiso funcional hepático, lo cual resulta complejo debido a las múltiples funciones y gran reserva fisiológica del hígado. Al no existir consenso acerca de cual es el mejor medio para este propósito, y con el fin de reportar y comparar el efecto del deterioro funcional en la morbilidad post operatoria, numerosos estudios utilizaron la clasificación de Child-Pugh (CP), para categorizar a los pacientes de acuerdo a su grado de daño hepático. Si bien esta clasificación ha sido ampliamente utilizada, presenta problemas como, por ejemplo, que los límites entre las categorías son arbitrarios, pudiendo existir diferencias importantes de compromiso funcional en una misma categoría, y subjetividad tanto en la determinación de los parámetros que considera, como en la asignación del puntaje que le corresponde al paciente en parámetros como nutrición y ascitis. El desarrollo de un modelo de regresión lineal, conocido como MELD, sigla en inglés de Model for End-Stage Liver Disease, desarrollado para tratar de predecir la mortalidad de pacientes cirróticos sometidos a un shunt portosistémico intrahepático transyugular $(\text { TIPS })^{18}$, identificó el $\log _{e}$ de las concentraciones de creatinina, bilirrubina, y del valor del INR como valores independientes predictores de sobrevida. Estos se relacionan en una fórmula matemática $\left(\mathrm{MELD}=3,78 \times \log _{\mathrm{e}}(\mathrm{Brr} \mathrm{mg} / \mathrm{dl})+\right.$ $\left.11,2 \times \log _{e}(\mathrm{INR})+9,57 \times \log _{e}(\mathrm{Cr} \mathrm{mg} / \mathrm{dl})+6,43\right)$, cuyo resultado se obtiene fácilmente accediendo a http://www.mayoclinic.org/meld/mayomodel5. html. Los valores de MELD varían entre 6 y 40, dando un rango más amplio que los 5 y 15 puntos de la clasificación de $\mathrm{CP}$, permitiendo una mejor diferenciación en el grado de compromiso hepático de cada paciente. MELD ofrece la posibilidad de utilizar un método simple, objetivo, fácil de 
aplicar, que evita los problemas de subjetividad de la clasificación de CP. Entre las posibles ventajas de MELD estaría el permitir uniformar la manera de clasificar y cuantificar el riesgo, y más que establecer categorías de este, relacionar aumentos del puntaje con aumentos del riesgo. Estas ventajas han hecho que en USA sea utilizado desde 2002 para la ubicación de pacientes en lista de espera de trasplante, y para la determinación de riesgo de paciente sometidos a cirugía ${ }^{6-9,19,20}$. La utilización del score MELD, en distintas situaciones clínicas, ha mostrado que este es al menos equivalente, y en otras situaciones superior al CP para determinar el riesgo de estos pacientes ${ }^{17,18,21-23}$.

\section{Riesgo y sus determinantes}

Considerando que la literatura muestra que en estos pacientes puede realizarse prácticamente todo tipo de cirugía, debemos conocer cuales son los riesgos que enfrentan. La morbilidad se relaciona por una parte con el deterioro adicional de la función hepática, manifestándose como aparición o aumento de ascitis, encefalopatía, sangramiento digestivo, insuficiencia renal e insuficiencia hepática ${ }^{17}$. Por otro lado, se relaciona con la aparición de complicaciones infecciosas, especialmente respiratorias $^{5}$ y sépticas acompañadas de falla multiorgánica, siendo ésta en algunas series la principal causa de muerte ${ }^{3}$. La Tabla 1 muestra la morbilidad y mortalidad encontrada en algunos de los estudios revisados. Las diferencias observadas se explican por diferencias entre los grupos estudiados respecto a compromiso de función hepática, definiciones utilizadas, tipo de cirugía, experiencia quirúrgica, etc. Estas diferencias hacen evidente la necesidad de tratar de precisar la importancia de los posibles factores de riesgo presentes en el paciente que se está evaluando:

Tipo de cirugía: Northup ${ }^{6}$, analizando pacientes con $\mathrm{CH}$ sometidos a procedimientos quirúrgicos intraabdominales, ortopédicos y cardiovasculares, encontró que la cirugía extra abdominal y extra torácica se acompaña de una menor morbimortalidad. En este estudio, la cirugía intraabdominal presentó una mortalidad a 30 días de 23,9\%, siendo el score MELD el único predictor de mortalidad. Buscando determinar el riesgo en distintos momentos del post operatorio, Teh ${ }^{8}$ estudió 772 pacientes con $\mathrm{CH}$ sometidos a cirugía digestiva, ortopédica, y cardiovascular. Los resultados mostraron que a partir de valores de 8 de MELD existe una relación casi lineal con riesgo relativo, siendo MELD el mejor predictor de mortalidad a $30 \mathrm{y}$ 90 días. Probablemente lo más significativo de este estudio es la capacidad de poder determinar, basado en MELD, el riesgo de mortalidad en momentos específicos del post operatorio, con lo cual se haría una estimación más fundamentada del riesgo que afronta cada paciente. Se puede acceder a este cálculo de riesgo a través del sitio web citado previamente. Aún cuando este estudio aparece muy atractivo para estimar riesgo, debe mencionarse que su utilidad pudiera verse limitada por tratarse de un estudio retrospectivo, con pacientes tratados en un solo centro, y con la posibilidad de selección de los pacientes que fueron operados, lo que podría limitar su generalización. Además podría no ser representativo del riesgo de pacientes con MELD mayor de 15, ya que sólo 58 de los 772 pacientes estudiados tenían este puntaje, y podría subestimar el riesgo de los pacientes sometidos a cirugía cardiovascular, el cual ha sido reportado como mayor en otras series ${ }^{13,24-26}$.

Patología agregada: La presencia de insuficiencia cardíaca, y creatinina elevada se han demostrado asociadas a una mayor mortalidad ${ }^{5,6,27}$.

Urgencia: Ha sido identificada como un factor independiente determinante de mayor riesgo de morbimortalidad ${ }^{4,5,8,15,23}$. En análisis multivariables la condición de urgencia pudiera no demostrarse asociado a mayor mortalidad debido a que los pacientes operados de urgencia presentan score MELD mayores, que reflejan un mayor deterioro de la función renal y hepática ${ }^{8}$.

\section{Tipos específicos de cirugía y riesgo}

\section{Cirugía biliar}

En cirugía biliar, la colecistectomía laparoscópica ha mostrado ventajas como disminución de la morbilidad, menores requerimientos de transfusión, y una menor incidencia de complicaciones asociadas a la herida operatoria ${ }^{16,28-30}$. Una menor duración de la hospitalización no ha sido uniformemente demostrada ${ }^{16,31}$. En relación a pacientes sin co morbilidad, un meta análisis reciente $^{10}$, confirma la mayor morbilidad de los pacientes con $\mathrm{CH}(20,8 \mathrm{v} / \mathrm{s} 7,9 \%)$, y muestra que 
Tabla 1. Morbilidad y mortalidad perioperatorias en cirrosis

\begin{tabular}{|c|c|c|c|c|}
\hline Autor & Cirugía & Morbilidad & $\begin{array}{l}\text { Mortalidad } \\
\text { (30 días) }\end{array}$ & Comentario \\
\hline Northup ${ }^{6}$ & $\begin{array}{l}\text { Abdominal, músculo } \\
\text { esquelética, cardio- } \\
\text { vascular, otras }\end{array}$ & & $\begin{array}{l}23,9 \% \text { (abdominal) } \\
16,4 \% \text { (global) }\end{array}$ & \\
\hline Garrison $^{3}$ & Abdominal & & $\begin{array}{l}\text { Child A } 10 \% \\
\text { Child B } 31 \% \\
\text { Child C } 76 \%\end{array}$ & \\
\hline Mansour ${ }^{4}$ & Abdominal & $\begin{array}{l}\text { Child A 20,8\% } \\
\text { Child B } 33 \% \\
\text { Child C } 36 \%\end{array}$ & $\begin{array}{l}18 \% \text { electivos } \\
50 \% \text { urgencia }\end{array}$ & $\begin{array}{l}\text { No define período en que se } \\
\text { estudia mortalidad }\end{array}$ \\
\hline Del Olmo ${ }^{17}$ & Abdominal & $50,4 \%$ & $16,3 \%$ & \\
\hline Jakab21 & Abdominal & $97 \%$ & $\begin{array}{l}\text { Electivo } 17 \% \\
\text { Urgencia } 19 \%\end{array}$ & $\begin{array}{l}\text { Alto porcentage de pacientes } \\
\text { Child B y C, y cirugías de alto } \\
\text { riesgo }\end{array}$ \\
\hline Ziser $^{5}$ & $\begin{array}{l}\text { Abdominal, vascular, } \\
\text { urinaria, ortopédica, } \\
\text { otorrino, oftalmológica }\end{array}$ & $30,1 \%$ & $11,6 \%$ & $\begin{array}{l}\text { Incluye alto porcentage de } \\
\text { cirugías de bajo riesgo }\end{array}$ \\
\hline Aranha ${ }^{15}$ & Colecistectomía abierta & & $\begin{array}{l}86 \% \text { urgencia } \\
41 \% \text { electivo }\end{array}$ & \\
\hline Fernandes ${ }^{16}$ & $\begin{array}{l}\text { Colecistectomía } \\
\text { laparoscópica }\end{array}$ & $12,5 \%$ & 0 & No incluye pacientes Child C \\
\hline Puggioni ${ }^{10}$ & $\begin{array}{l}\text { Colecistectomía } \\
\text { laparoscópica }\end{array}$ & $20,9 \%$ & 0 & $\begin{array}{l}\text { Metaanálisis, incluye sólo } 6 \\
\text { pacientes Child C }\end{array}$ \\
\hline Teh $^{32}$ & Resección hepática & & $\begin{array}{l}\text { MELD } \leq 8,0 \% \\
M E L D \geq 9,29 \%\end{array}$ & \\
\hline Cuchetti $^{9}$ & Resección hepática & $\begin{array}{l}\text { MELD } \leq 9,8,1 \% \\
\text { MELD } 9-10,35,7 \% \\
M E L D \geq 11,83,3 \%\end{array}$ & & $\begin{array}{l}\text { Falla hepática y MELD: } \\
<9,0 \% \\
9-10,3,6 \% \\
\geq 11,37,5 \%\end{array}$ \\
\hline Hayashida ${ }^{25}$ & Coronaria y valvular & $\begin{array}{l}\text { Child A 6/10 } \\
\text { Child B } 7 / 7 \\
\text { Child C } 1 / 1\end{array}$ & $\begin{array}{l}\text { Child A 0/10 } \\
\text { Child B 2/4 } \\
\text { Child C } 1 / 1\end{array}$ & \\
\hline Kaplan'24 & Coronaria y valvular & & $\begin{array}{l}\text { Global 30\% } \\
\text { Child B 50\% }\end{array}$ & \\
\hline $\operatorname{Lin}^{14}$ & Coronaria y valvular & $\begin{array}{l}\text { Child A } 39 \% \\
\text { Child B y C } 80 \%\end{array}$ & 1/18 (Child A) & $\begin{array}{l}\text { Muy discutible, concluye que } \\
\text { cirugía es segura en pacientes } \\
\text { con } \mathrm{CH}\end{array}$ \\
\hline Bizouarn 26 & Coronaria y valvular & 7 de 10 pacientes & 1 de 10 & \\
\hline
\end{tabular}

estos además presentan una mayor tasa de conversión cuando se someten a cirugía laparoscópica, un mayor tiempo operatorio, mayores complicaciones hemorrágicas, y una menor duración de la hospitalización que cuando se realiza cirugía abierta. Debe mencionarse sin embargo, que en este estudio sólo se incluyen 6 pacientes Child
C. Si bien la impresión general es que la cirugía laparoscópica parecería ser beneficiosa en estos pacientes, el mismo estudio señala deben realizarse estudios prospectivos que determinen el real efecto de la cirugía laparoscópica, especialmente en pacientes Child C. Conclusiones y problemas similares muestra una casuística nacional ${ }^{12}$. 


\section{Cirugía resectiva hepática}

En cirugía resectiva hepática, a los problemas asociados a la disminución de flujo hepático determinados por la anestesia y la cirugía, se agrega la disminución del parénquima funcional. Al igual que en otros tipos de cirugía, existe consenso que un mayor deterioro pre operatorio de la función hepática se acompaña de una mayor morbimortalidad $^{7,9,32-35}$. Si bien hay estudios que muestran que la clasificación de CP puede ser útil en la evaluación funcional de pacientes que requieren resección hepática ${ }^{36-38}$, MELD parece ser más preciso en identificar pacientes de alto riesgo. A pesar de esto, no existe consenso acerca de los límites para la realización de una resección hepática ${ }^{9,32}$. La aparentemente buena capacidad predictiva de morbimortalidad del score MELD en cirugía resectiva hepática no ha sido uniformemente encontrada. Schroeder ${ }^{7}$ encontró que la clasificación de la American Society of Anesthesiology (ASA), y de $\mathrm{CP}$ eran mejores predictores de morbimortalidad post resección. El hecho que los estudios de Teh y Cuchetti ${ }^{9,32}$ reporten pacientes con MELD promedio de 9, contra 6,5 de los pacientes de Schroeder podría explicar estas diferencias. Teh además encontró que a corto plazo, la clínica tumoral y la clasificación de ASA son buenos predictores de mortalidad, mientras que a largo plazo lo son el tamaño tumoral $(>5 \mathrm{~cm})$, grado del tumor, y la ausencia de cápsula. Aún cuando el número de pacientes en este estudio es relativamente limitado, la importancia de MELD y del tamaño tumoral ha sido también encontrado en otros estudios ${ }^{33,38,39}$.

\section{Cirugía cardiovascular}

La cirugía cardíaca en estos pacientes presenta un muy elevado riesgo de morbimortalidad (Tabla 1). Se han reportado pacientes con $\mathrm{CH}$ candidatos a TH y enfermedad coronaria con indicación quirúrgica, no existiendo respuesta acerca de cual patología debe ser resuelta primero ${ }^{40}$. El riesgo de deterioro adicional de la función hepática en pacientes con compromiso previo de esta es un hecho real. En esto pueden influir los cambios en la magnitud y tipo de flujo hepático asociados a la circulación extracorpórea (CEC), sin embargo, los factores más importantes determinantes de disfunción hepática post CEC, parecen ser la condición hemodinámica pre y post operatoria, y el estado nutricional ${ }^{41}$. Las casuísticas reportadas son todas pequeñas y retrospectivas ${ }^{13,14,24-26}$. Con excepción de un estudio que presenta una conclusión muy cuestionable $^{14}$, hay consenso que se trata de una cirugía de muy alta morbimortalidad, claramente relacionada con el grado de deterioro de función hepática. Por este motivo se sugiere que la indicación quirúrgica debiera limitarse a pacientes clasificados como Child A.

El momento en el cual debe realizarse la cirugía, también es controversial, no existiendo una respuesta definitiva al respecto. De acuerdo a Teh y colaboradores, MELD mayor de 20 se acompañan de una mortalidad tan alta que hacen planteable la posibilidad que procedimientos electivos se realicen posterior a un $\mathrm{TH}^{8}$, o incluso en situaciones específicas, como pacientes que han requerido revascularización miocárdica, en forma combinada con el $\mathrm{TH}^{40}$. No existe consenso sin embargo, acerca del puntaje MELD con el cual una cirugía electiva debiera ser postergada. Factores como la experiencia quirúrgica y la disponibilidad de órganos en cada lugar son importantes factores a considerar ${ }^{8}$. Si bien no existe acuerdo acerca del puntaje MELD que representa un riesgo demasiado elevado para la cirugía, esta sería relativamente segura con MELD menores de 10, aumentando dramáticamente cuando este es mayor de $20^{6}$.

Aún cuando es razonable plantear que la corrección de los factores de riesgo presentes se debería acompañar de una reducción de la morbimortalidad, no hay estudios que así lo demuestren.

De lo hasta aquí comentado, resulta evidente que, a excepción de procedimientos resectivos hepáticos, pacientes con $\mathrm{CH}$, incluso con deterioro avanzado de función hepática, pueden someterse a cualquier cirugía. Sin embargo, el riesgo de morbimortalidad puede ser considerado prohibitivo por el paciente y el equipo médico. Aún cuando la utilización de MELD como índice pronóstico ha incorporado elementos objetivos en la evaluación de estos pacientes, la decisión final de proceder con la cirugía seguirá siendo individual, dependiendo de la discusión informada con el paciente y/o su familia, de los riesgos específicos del procedimiento planificado en relación al grado de compromiso funcional hepático, y de la experiencia del equipo tratante.

\section{Referencias}

1. Propst A, Propst T, Zangerl G, Ofner D, Judmaier G, Vogel W. Prognosis and life expectancy in chronic liver disease. Dig Dis Sci 1995; 40: 1805-15. 
2. Gelman SI. Disturbances in hepatic blood flow during anesthesia and surgery. Arch Surg 1976; 111: 881-3.

3. Garrison RN, Cryer HM, Howard DA, Polk HC, Jr. Clarification of risk factors for abdominal operations in patients with hepatic cirrhosis. Ann Surg 1984; 199: 648-55.

4. Mansour A, Watson W, Shayani V, Pickleman J. Abdominal operations in patients with cirrhosis: still a major surgical challenge. Surgery 1997; 122: 730-5; discussion 5-6.

5. Ziser A, Plevak DJ, Wiesner RH, Rakela J, Offord KP, Brown DL. Morbidity and mortality in cirrhotic patients undergoing anesthesia and surgery. Anesthesiology 1999; 90: 42-53.

6. Northup PG, Wanamaker RC, Lee VD, Adams RB, Berg CL. Model for End-Stage Liver Disease (MELD) predicts nontransplant surgical mortality in patients with cirrhosis. Ann Surg 2005; 242: 244-51.

7. Schroeder RA, Marroquin CE, Bute BP, Khuri S, Henderson WG, Kuo PC. Predictive indices of morbidity and mortality after liver resection. Ann Surg 2006; 243: 373-9.

8. Teh SH, Nagorney DM, Stevens SR, Offord KP, Therneau TM, Plevak DJ, et al. Risk factors for mortality after surgery in patients with cirrhosis. Gastroenterology 2007; 132: 1261-9.

9. Cucchetti A, Ercolani G, Vivarelli M, Cescon M, Ravaioli M, La Barba G, et al. Impact of model for end-stage liver disease (MELD) score on prognosis after hepatectomy for hepatocellular carcinoma on cirrhosis. Liver Transpl 2006; 12: 966-71.

10. Puggioni A, Wong LL. A metaanalysis of laparoscopic cholecystectomy in patients with cirrhosis. J Am Coll Surg 2003;197:921-6.

11. Ji W, Li LT, Wang ZM, Quan ZF, Chen XR, Li JS. A randomized controlled trial of laparoscopic versus open cholecystectomy in patients with cirrhotic portal hypertension. World J Gastroenterol 2005; 11: 2513-7.

12. Poniachik J, Castro S, Madrid AM, Quera R, Amat J, Smok G, et al. Colecistectomía laparoscópica clásica en pacientes con cirrosis hepática. Rev Med Chile 2002; 130: $1343-8$

13. Klemperer JD, Ko W, Krieger KH, Connolly M, Rosengart TK, Altorki NK, et al. Cardiac operations in patients with cirrhosis. Ann Thorac Surg 1998; 65: 85-7.

14. Lin CH, Lin FY, Wang SS, Yu HY, Hsu RB. Cardiac surgery in patients with liver cirrhosis. Ann Thorac Surg 2005; 79: 1551-4.

15. Aranha GV, Sontag SJ, Greenlee HB. Cholecystectomy in cirrhotic patients: a formidable operation. Am J Surg 1982; 143: 55-60.
16. Fernandes NF, Schwesinger WH, Hilsenbeck SG, Gross GW, Bay MK, Sirinek KR, et al. Laparoscopic cholecystectomy and cirrhosis: a case-control study of outcomes. Liver Transpl 2000; 6: 340-4.

17. del Olmo JA, Flor-Lorente B, Flor-Civera B, Rodríguez F, Serra MA, Escudero A, et al. Risk factors for nonhepatic surgery in patients with cirrhosis. World J Surg 2003; 27: 647-52.

18. Malinchoc M, Kamath PS, Gordon FD, Peine CJ, Rank J, ter Borg PC. A model to predict poor survival in patients undergoing transjugular intrahepatic portosystemic shunts. Hepatology 2000; 31: 864-71.

19. O'Leary JG, Friedman LS. Predicting surgical risk in patients with cirrhosis: from art to science. Gastroenterology 2007; 132: 1609-11.

20. Wiesner R, Edwards E, Freeman R, Harper A, Kim R, Kamath $\mathrm{P}$, et al. Model for end-stage liver disease (MELD) and allocation of donor livers. Gastroenterology 2003; 124: 91-6.

21. Jakab F, Rath Z, Sugar I, Ledniczky G, Faller J. Complications following major abdominal surgery in cirrhotic patients. Hepatogastroenterology 1993; 40: 176-9.

22. Forman LM, Lucey MR. Predicting the prognosis of chronic liver disease: an evolution from child to MELD. Mayo End-stage Liver Disease. Hepatology 2001; 33: 473-5.

23. Farnsworth N, Fagan SP, Berger DH, Awad SS. ChildTurcotte-Pugh versus MELD score as a predictor of outcome after elective and emergent surgery in cirrhotic patients. Am J Surg 2004; 188: 580-3.

24. Kaplan M, Cimen S, Kut MS, Demirtas MM. Cardiac operations for patients with chronic liver disease. Heart Surg Forum 2002; 5: 60-5.

25. Hayashida N, Shoujima T, Teshima H, Yokokura Y, Takagi K, Tomoeda H, et al. Clinical outcome after cardiac operations in patients with cirrhosis. Ann Thorac Surg 2004; 77: 500-5.

26. Bizouarn P, Ausseur A, Desseigne P, Le Teurnier Y, Nougarede B, Train M, et al. Early and late outcome after elective cardiac surgery in patients with cirrhosis. Ann Thorac Surg 1999; 67: 1334-8.

27. Rice HE, O'Keefe GE, Helton WS, Johansen K. Morbid prognostic features in patients with chronic liver failure undergoing nonhepatic surgery. Arch Surg 1997;132:880-4; discussion 4-5.

28. Yerdel MA, Koksoy C, Aras N, Orita K. Laparoscopic versus open cholecystectomy in cirrhotic patients: a prospective study. Surg Laparosc Endosc 1997; 7: 483-6.

29. Saeki H, Korenaga D, Yamaga H, Mawatari K, Orita H, Itasaka $\mathrm{H}$, et al. A comparison of open and laparoscopic cholecystectomy for patients with cirrhosis. Surg Today 1997; 27: 411-3. 
30. Poggio JL, Rowland CM, Gores GJ, Nagorney DM, Donohue JH. A comparison of laparoscopic and open cholecystectomy in patients with compensated cirrhosis and symptomatic gallstone disease. Surgery 2000; 127: 405-11.

31. Jan YY, Chen MF. Laparoscopic cholecystectomy in cirrhotic patients. Hepatogastroenterology 1997; 44: 1584-7.

32. Teh SH, Christein J, Donohue J, Que F, Kendrick M, Farnell M, et al. Hepatic resection of hepatocellular carcinoma in patients with cirrhosis: Model of End-Stage Liver Disease (MELD) score predicts perioperative mortality. J Gastrointest Surg 2005; 9: 1207-15; discussion 15.

33. Belghiti J, Regimbeau JM, Durand F, Kianmanesh AR, Dondero F, Terris B, et al. Resection of hepatocellular carcinoma: a European experience on 328 cases. Hepatogastroenterology 2002; 49: 41-6.

34. Bruix J, Castells A, Bosch J, Feu F, Fuster J, GarcíaPagan JC, et al. Surgical resection of hepatocellular carcinoma in cirrhotic patients: prognostic value of preoperative portal pressure. Gastroenterology 1996; 111: 1018-22.

35. Nagasue N, Kohno H, Tachibana M, Yamanoi A, Ohmori H, El-Assal ON. Prognostic factors after hepatic resection for hepatocellular carcinoma associated with Child-Turcotte class B and C cirrhosis. Ann Surg 1999;
229: 84-90.

36. Franco D, Capussotti L, Smadja C, Bouzari H, Meakins J, Kemeny F, et al. Resection of hepatocellular carcinomas. Results in 72 European patients with cirrhosis. Gastroenterology 1990; 98: 733-8.

37. Paquet KJ, Gad HA, Lazar A, Koussouris P, Mercado MA, Heine WD, et al. Analysis of factors affecting outcome after hepatectomy of patients with liver cirrhosis and small hepatocellular carcinoma. Eur J Surg 1998; 164: 513-9.

38. Wayne JD, Lauwers GY, Ikai I, Doherty DA, Belghiti J, Yamaoka Y, et al. Preoperative predictors of survival after resection of small hepatocellular carcinomas. Ann Surg 2002; 235: 722-30; discussion 30-1.

39. Poon RT, Fan ST, Lo CM, Ng IO, Liu CL, Lam CM, et al. Improving survival results after resection of hepatocellular carcinoma: a prospective study of 377 patients over 10 years. Ann Surg 2001; 234: 63-70.

40. Morris JJ, Hellman CL, Gawey BJ, Ramsay MA, Valek TR, Gunning TC, et al. Case 3-1995. Three patients requiring both coronary artery bypass surgery and orthotopic liver transplantation. J Cardiothorac Vasc Anesth 1995; 9: 322-32.

41. Hild P. Pathophysiology of cardiopulmonary bypass. In: Henssley FA MD, ed. The practice of cardiac Anesthesia Boston: Little, Brown 1990; 617. 